\title{
Current Activity on the Martian Surface: A Key Subject for Future Exploration
}

A White Paper Submitted to the Planetary Science and Astrobiology Decadal Survey 2023-2032

Corresponding Author:

Colin M. Dundas

U.S. Geological Survey, Astrogeology Science Center

2255 N. Gemini Dr., Flagstaff, AZ, 86001

cdundas@usgs.gov

Co-authors: Shane Byrne ${ }^{1}$, Matthew Chojnacki ${ }^{2}$, Serina Diniega ${ }^{3}$, Ingrid J. Daubar ${ }^{4}$, Christopher W. Hamilton ${ }^{1}$, Candice J. Hansen ${ }^{2}$, Alfred S. McEwen ${ }^{1}$, Ganna Portyankina ${ }^{5}$, Hanna G.

Sizemore ${ }^{2}$

Signatories: Chimira Andres ${ }^{6}$, Mariah M. Baker ${ }^{7}$, Jonathan Bapst ${ }^{3}$, Gwendolyn Bart ${ }^{8}$, Nicole F. Baugh $^{1}$, Nicole M. Bardabelias ${ }^{1}$, Ross A. Beyer ${ }^{9,10}$, Ali M. Bramson ${ }^{1,11}$, Veronica J. Bray ${ }^{1}$, Peter B. Buhler ${ }^{3}$, Nathalie A. Cabrol' ${ }^{9}$, Camila Cesar ${ }^{12}$, Susan J. Conway ${ }^{13}$, Jay L. Dickson ${ }^{14}$, Cynthia L. Dinwiddie ${ }^{15}$, Lori K. Fenton ${ }^{9}$, Tanya N. Harrison ${ }^{16}$, Paul O. Hayne ${ }^{5}$, Ernst Hauber ${ }^{17}$, James W. Head III ${ }^{4}$, Kenneth E. Herkenhoff ${ }^{18}$, Thomas Heyer ${ }^{19}$, Shannon M. Hibbard ${ }^{6}$, Christine S. Hvidberg $^{20}$, Devanshu Jha ${ }^{21}$, Mikhail A. Kreslavsky ${ }^{22}$, Margaret E. Landis ${ }^{5}$, Sylvain Piqueux ${ }^{3}$, Jan Raack ${ }^{19}$, Christian J. Schaller ${ }^{1}$, Simone Silvestro ${ }^{23}$, Isaac B. Smith ${ }^{2,24}$, David E. Stillman ${ }^{15}$, Robert Sullivan ${ }^{25}$, Sarah S. Sutton ${ }^{1}$, Singleton Thibodeaux-Yost ${ }^{1}$, Daniela Tirsch ${ }^{17}$, Nicolas Thomas $^{12}$, Adomas Valantinas ${ }^{12}$, James J. Wray ${ }^{26}$

${ }^{1}$ University of Arizona, ${ }^{2}$ Planetary Science Institute, ${ }^{3}$ Jet Propulsion Laboratory, ${ }^{4}$ Brown University, ${ }^{5}$ University of Colorado, ${ }^{6}$ University of Western Ontario, ${ }^{7}$ Smithsonian Institution, ${ }^{8}$ University of Idaho, ${ }^{9}$ SETI Institute, ${ }^{10}$ NASA Ames Research Center, ${ }^{11}$ Purdue University, ${ }^{12}$ Universität Bern, ${ }^{13}$ Laboratoire de Planétologie et Géodynamique CNRS, ${ }^{14}$ California Institute of Technology, ${ }^{15}$ Southwest Research Institute, ${ }^{16}$ Planet Labs, ${ }^{17}$ DLR, ${ }^{18}$ U.S. Geological Survey, ${ }^{19}$ Westfälische Wilhelms-Universität, ${ }^{20}$ University of Copenhagen, ${ }^{21}$ MVJCE, ${ }^{22}$ University of California Santa Cruz, ${ }^{23} \mathrm{INAF},{ }^{24}$ York University, ${ }^{25}$ Cornell University, ${ }^{26} \mathrm{Georgia}$ Institute of Technology.

Acknowledgements: A portion of the research was carried out at the Jet Propulsion Laboratory, California Institute of Technology, under a contract with the National Aeronautics and Space Administration (80NM0018D0004). 


\section{Overview}

One of the fundamental discoveries in Mars science in the last decade has been the extent and importance of current surface activity. Recent results have shifted our view of Mars from a world where the most interesting geologic events were in the distant past (similar to the Moon) to a world that undergoes active evolution and one where understanding the present is key to deciphering the planet's history. When input was requested for the last Planetary Science Decadal Survey, some observations of surface changes had been published, but the number of detections was small and their significance not fully appreciated. Since that time, detections have proliferated, driven primarily by the long-term operation of the Mars Reconnaissance Orbiter (MRO) and the High Resolution Imaging Science Experiment (HiRISE) as well as landed observations of aeolian activity. In addition to observed changes, theory suggests that additional important surface processes are likely active, but not yet observed because orbital data are limited in space and time and landed studies are rare.

Understanding current Martian surface processes is a fundamental science question in itself, as it provides a test for physical and terrestrial analog-based models of specific geological processes acting under extraterrestrial planetary and environmental conditions. It is also an essential step for reading Mars' geologic history and providing input to climate models: without understanding current dynamic processes, we cannot understand how they have varied during recent climate cycles, nor how they are reflected in ancient rock or modern ice records. Understanding the rates and types of current surface activity is also highly relevant to selecting geological samples, setting Planetary Protection rules, and understanding the hazards and environment that would be experienced by future human explorers.

\section{State of Knowledge}

This section describes many different types of observed surface changes and their likely causes. Overviews and seminal studies are cited, but this is not a comprehensive review.

Recurring Slope Lineae (RSL; Fig. 1A) have been one of the most puzzling discoveries [1] in Mars science since the last Decadal Survey. RSL are flows that recur each Mars year in approximately the same location. They are darker than their surroundings and gradually grow downhill. Interpretations of these dark streaks initially favored flowing liquid water or brine at the surface of Mars today. Subsequent hypotheses based on flow of dry sand or dust have emerged [2], but the community has not yet reached consensus. If not caused by liquid flow, then RSL may indicate Martian processes with no terrestrial analogs, but which can teach us about geomorphological processes active on worlds more similar to Mars than Earth.

Early studies interpreted gullies (Fig. 1B-C) as evidence for flowing water within the last several million years and perhaps today [3, 4]. At the time of the last Decadal Survey, the leading interpretation was that gullies formed via melting snow or near-surface ice $[5,6]$. The discovery of widespread activity called this hypothesis into question: gully flows are common and strongly correlate with the occurrence of $\mathrm{CO}_{2}$ frost, although the flow processes are not yet well understood [7]. So-called "linear" dune gullies are also active, with present-day changes apparently driven by sliding blocks of $\mathrm{CO}_{2}$ frost [8]. Current changes suggest that non-aqueous processes are major contributors to gully formation and open the possibility that gullies form entirely by $\mathrm{CO}_{2}$ frost processes. Gully formation processes are thus a critical open question which must be answered to interpret recent climate variations. 

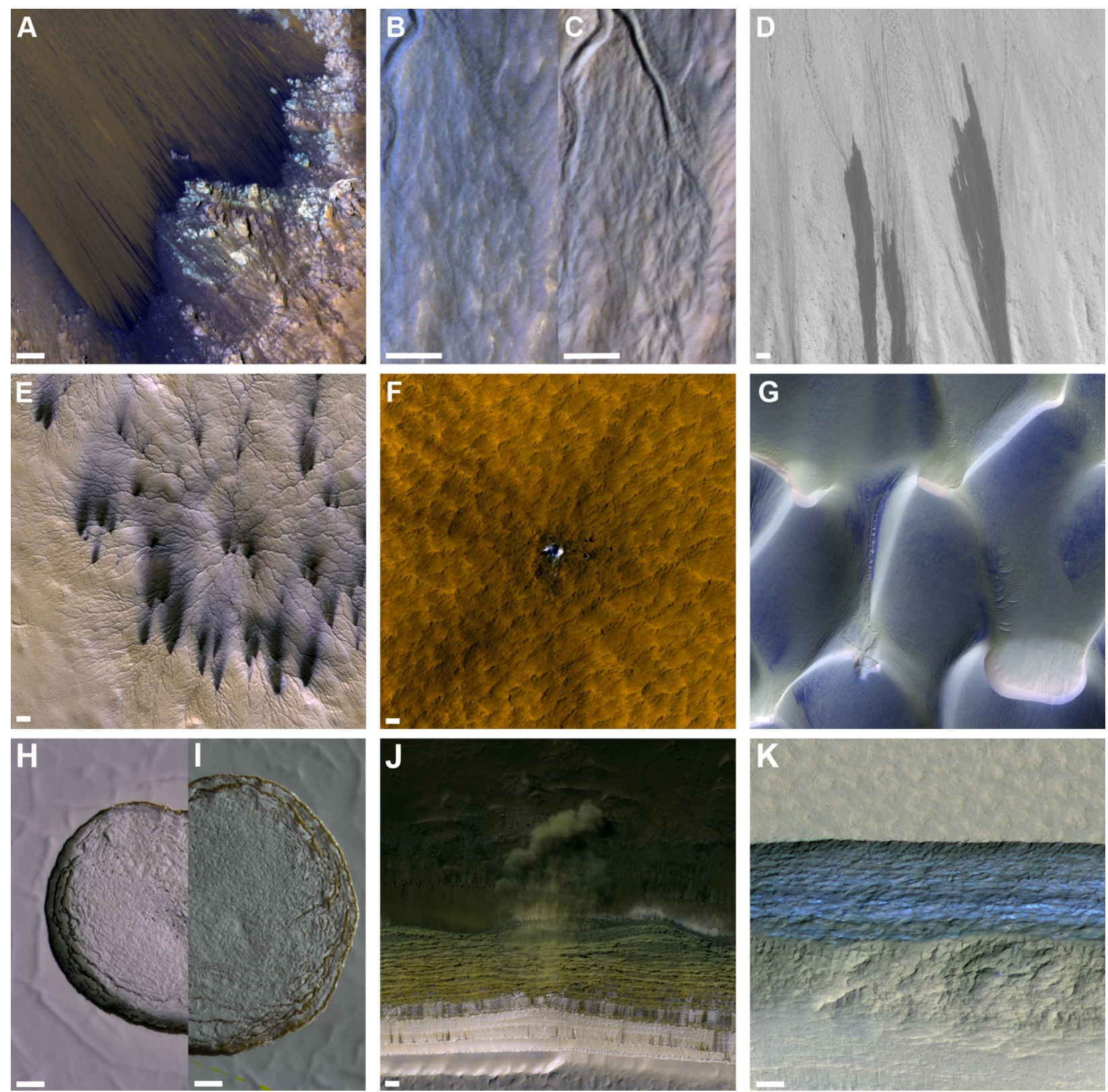

Figure 1: Example active surface features on Mars. A: Recurring Slope Lineae. B, C: Gully change with channel incision. D: Slope streak with rockfall tracks. E: Araneiform with seasonal fans. F: New impact crater exposing ground ice. G: Active sand dunes with new alcoves. H, I: Growth of pit in $\mathrm{CO}_{2}$ South Polar Residual Cap over six Mars years. J: Avalanche at the edge of the North Polar Layered Deposits. K: Icy scarp undergoing sublimation. HiRISE images courtesy NASA/JPL/University of Arizona. Scale bars are all $25 \mathrm{~m}$.

Additional slope processes are occurring planet wide. These include slope streaks (Fig. 1D), which are the subject of a wet-versus-dry controversy that parallels the RSL debate $[9,10]$. Slope streaks are also darker than their surroundings, but form on bright dusty slopes and do not grow gradually [11]. New rockfalls and short of high-latitude rocks have been observed [12], but the driving processes are uncertain. Avalanches (Fig. 1J) and block falls on steep north polar scarps occur each year in a narrow seasonal window [13]. Repeated observations constrain scarp erosion rates and the overall history of the North Polar Layered Deposits [14]. 
Mars' $\mathrm{CO}_{2}$ atmosphere is in vapor pressure equilibrium with surface ice [15] and the yearly condensation and sublimation of $\mathrm{CO}_{2}$ at each pole leads to a wealth of seasonal processes. Spring is a particularly active time, with features identified first in Mars Orbiter Camera images including fan-shaped deposits on the seasonal polar caps and dendritic troughs dubbed "spiders" carved in the surface (Fig. 1E) [16-18], and active erosion discovered by HiRISE, such as new spiders [19] and alcoves on dunes (Fig. 1G) [19, 20]. Spider formation appears to be abetted by the presence of sand, but a longer observation time period may enable detection of slower erosion processes. This suite of processes and landforms has no terrestrial analog but is fundamentally important to Martian polar/climate science.

Perennial surface ices on Mars are in a stability regime where observable changes are possible over the course of a spacecraft mission and diagnostic of surface environmental conditions. "Swiss Cheese" pits within perennial polar $\mathrm{CO}_{2}$ ice are evolving in shape and size by up to several meters per Martian year (Fig. 1H-I) [21]. As well as these year-to-year changes, the south polar residual $\mathrm{CO}_{2}$ cap shows extensive refrosting in response to global dust storms through unclear mechanisms. Better understanding of this contemporary activity would help characterize past climates that are recorded in large buried $\mathrm{CO}_{2}$ ice deposits [22] and other stratigraphic units.

Water ice deposits change more slowly than $\mathrm{CO}_{2}$ ice, yet changes in the perennial north polar water ice cap [23], and sublimation of exposed ice (Fig. 1K) [24, 25] have both been observed and inform estimates of near-surface water vapor and ice properties. Evolution of both the subsurface ice table and associated landforms are likely ongoing [26, 27], at rates too slow to observe with current methods. However, some fundamental observables are not well determined (e.g., we do not know whether the perennial $\mathrm{CO}_{2}$ and $\mathrm{H}_{2} \mathrm{O}$ caps are presently gaining or losing mass [28]).

At the time of the last Decadal Survey, only a handful of new impacts had been discovered on Mars, with formation dates constrained by before-and-after images [4]. Since then, MRO has discovered over a thousand [29, 30]. This has spurred studies of their statistics and implications for Martian crater chronology, morphology, and aeolian modification. These new impacts allow for studies of not only impact cratering processes (a physical process ubiquitous in the Solar System but that is difficult to reproduce in a laboratory or numerical models), but also atmospheric fragmentation processes, dust settling rates, weathering processes that occur at fresh exposures of the shallow subsurface, and high energy atmosphere-surface interactions, all of which are not yet well understood. Additionally, some impact craters expose subsurface ice (Fig. 1F) [24], providing information about the properties and global distribution of shallow ice.

The last decade of Mars exploration has revealed that current atmospheric winds are capable of frequently transporting surface sediment at large scales in the form of moving sand ripples and dunes [31-33]. Global studies have shown widespread, geographically variable bedform activity and quantified volumetric sand fluxes based on repeat high-resolution orbital images and topography [34]. The period with the most geomorphically-effective winds in Nili Patera, as inferred from high sediment fluxes, occurs during maximum atmospheric solar heating and peak atmospheric pressure at perihelion [35]. North polar ground ice and seasonal frost limit winddriven changes of the circumpolar ergs to the warmest portions of northern spring and summer, while seasonal $\mathrm{CO}_{2}$ ice can promote higher sand avalanche fluxes for larger dunes [20, 36, 37]. In contrast, southern dune fields $\left(>45^{\circ} \mathrm{S}\right)$ are less mobile, perhaps as a result of longer and colder winters and lower surface pressure [38, 39]. Open questions persist regarding how aeolian bedforms respond to regional and global dust storms, as well as long-term rates of aeolian-driven landscape evolution, including how these processes interact with seasonal and perennial ices. 
Curiosity rover observations of decameter-scale active ripples in Gale crater indicate asymmetric profiles with angle-of-repose lee slopes and sinuous crest lines, unlike smaller terrestrial wind ripples [40]. These may be fluid-drag ripples [40], which on Earth occur under water, but on Mars could form by wind due to the low-density atmosphere. Another proposed mechanism suggests that "saltation clusters," which develop as sand particles are aerodynamically entrained at speeds between the impact and fluid velocity thresholds [41], form these meter-scale ripples [42]. Thus, observations of active aeolian features in a low-density atmosphere are providing new insights into the fundamentals of aeolian grain transport, applicable to many worlds.

A variety of other aeolian changes also occur planet-wide. These include regional albedo changes [43] as well as changes in wind streaks [44] and dust devil tracks [45].

Mars has only recently been confirmed to be active within its interior as well - the InSight (Interior Exploration using Seismic Investigations, Geodesy and Heat Transport) mission has detected signals from current seismic activity [46]. The first quakes to be observed have indicated a possible active seismic region near Cerberus Fossae, allowed an attempt at determining the crustal and upper mantle velocity structure, and revealed similarities and differences between Martian, lunar, and terrestrial seismicity, but geomorphic change has not yet been reported.

\section{Importance of Current Activity}

The study of active processes goes beyond an accumulation of lists of surface changes and quantification of their rates and effects. The fundamental objective is to understand the driving processes and thus improve interpretation of the specific features left by these processes on the Martian surface and in the stratigraphic record. Many of these processes were not predicted before their effects were observed and are not yet well understood.

Not understanding the processes currently active on Mars is a basic knowledge gap that prevents robust interpretation of the surface or connection of landforms to the current environment. Many forms of current activity have been surprising, based on terrestrial experience and the past perspectives of important planetary surface processes. For instance, the diverse processes and associated landforms driven by seasonal $\mathrm{CO}_{2}$ frost were unexpected and have no close terrestrial analog. Present-day sand transport was challenging for older theories to explain and led to suggestions of extreme wind speeds, but observations of activity have led us to understand the importance of bursts of saltation which require lower winds to maintain movement than were required to initiate movement. This example also demonstrates the close coupling between surface processes and the lower atmosphere. A new result is the coupling between dust distribution via regional and global dust storms and increased rates of subsequent spring surface sublimation activity [47] and dramatically higher rates of RSL formation [48]. Dust lifting, settling, and transport provides another fundamental linkage between surface and atmospheric processes [49]. In addition to understanding the current puzzles, it remains likely that present-day Mars has more unanticipated processes yet to be discovered.

The need to understand the modern surface is also essential to determining the climate of the recent past and reading the ancient rock record. Without a strong grasp on what processes are currently active and how they work, understanding the climate record is impossible. For instance, at the time of the last Decadal Survey, gullies were commonly thought to indicate melting snow within the last few million years [e.g. 5-6]. However, widespread current activity driven by $\mathrm{CO}_{2}$ frost raises the possibility that this is the sole recent gully-forming process and the recent climate was not conducive to snowmelt. These are important and widespread young landforms but their significance for past climate is thus uncertain. 
Present-day Martian processes with no terrestrial analog may also be represented in the ancient rock record and - without a clear understanding of these processes - such records may be missed or misinterpreted. Contemporary Martian aeolian activity results in bedforms without a close terrestrial analog, and frost is also a major agent of change on sand dunes. Both of these could produce stratification in Martian sandstones different from the patterns observed in terrestrial sandstones [40]. Similarly, $\mathrm{CO}_{2}$-driven activity in gullies produces deposits that from orbit appear similar to wet debris flows; how such deposits may appear in stratigraphy and whether such records can discriminate between water- and $\mathrm{CO}_{2}$ frost-driven past gully activity are open questions [7].

In addition to the direct value of understanding Martian processes, studies of surface change provide vital contextual information for other science objectives. For instance, observations of ice-exposing craters provide detailed local data on the ice content and overburden thickness, which can be used to corroborate and calibrate observations from synthetic-aperture radar (SAR) observations recommended by community studies [28, 50, 51] and recently suggested for a Mars Ice Mapper mission [52]. The debated role for liquid water for several common forms of activity also has potential implications for whether the current surface is habitable.

Current activity also directly impacts ongoing or future robotic and human exploration. Surface features such as RSL and mid-latitude gullies have been interpreted as indicators of liquid water and are thus regarded as "Uncertain" Special Regions [53]. Questions about their formation processes are the prime reason for this uncertainty. Sand and dust transport will affect astronauts' exposure to these materials and may drive abrasion or contamination of equipment [54], as well as control the distribution rate of wind- or grain-carried biological or biohazardous materials. Ongoing surface activity can also expose unaltered material from the subsurface. This may be particularly important for studying organics or volatiles, with implications for science investigations of past biomarkers or signs of habitability as well as future use of in situ resources.

\section{Recommended Future Measurements}

This section describes observations and measurements for the next decade that are needed to answer key knowledge gaps about current processes on Mars. The ultimate objective of the investigations outlined here is to understand the driving processes, and a variety of instruments and data types can contribute to reaching this goal. We do not focus on specific mission concepts but reference existing studies of implementation approaches when possible.

The bulk of currently known surface changes have been detected via orbital repeat images. Both the high resolution ( $\sim 0.3 \mathrm{~m} / \mathrm{pixel})$ and high signal-to-noise ratio (SNR) of HiRISE have proven uniquely valuable for change detection, plus MRO's ability to point for frequent off-nadir targeting which enables carefully targeted overlapping images. Sustaining such capabilities is of major importance for active-process studies as this provides the basic record of surface change. This points to two recommendations. First, continued operation of HiRISE with actively planned targeting campaigns is needed. Change-detection studies are inherently dynamic and require iterative, interactive planning. Second, by the end of the 2020s, a successor highresolution orbital imaging instrument is likely to be needed to replace this aging system, to observe active processes and plan future landed studies and exploration. $1 \mathrm{~m} /$ pixel or better orbital imaging, with repeated targeted imaging over a long temporal baseline, can detect many changes, but HiRISE-scale $(\sim 0.3 \mathrm{~m} / \mathrm{pixel})$ or better observations are needed to fully characterize changes from known processes. Complementing HiRISE-like imaging, context orbital imaging (CTXclass; $6 \mathrm{~m}$ /pix with extensive areal coverage) is valuable for detecting new impacts and broad-area changes. Higher SNR and multi-color imaging would improve context imaging, potentially 
improving the detection of new impacts in non-dusty regions [cf. 4, 29]. HiRISE- and CTX-scale imaging are fundamental to the detection and characterization of known active processes. Moreover, extending the temporal record has the potential to detect as-yet unknown active processes which are slow or rare. Additional orbital imaging from different or changing orbits could also reveal processes that occur at different times of day. MRO covers the dayside at $\sim 3$ PM and is blind to morning activity, although the Trace Gas Orbiter Colour and Stereo Surface Imaging System (CaSSIS) is beginning to address this issue. High-resolution stereo imaging capable of resolving changing topography is of high value to quantify rates of change, and for some processes, SAR interferometry or laser altimetry may allow detection of topographic changes. Highresolution spectral data would also provide useful information, particularly for frost detection.

In addition to the record of changes occurring, high-quality local environmental data are critical to understanding the causes of activity. Relevant meteorological data include surface and atmospheric temperature, pressure, wind speed and direction, and near-surface humidity within a few meters above the surface, as well as surface frost abundance, properties, and distribution [55]. Vertical resolution of atmospheric properties such as water vapor content and isotopic composition would also help understand surface-atmosphere linkages. Geologic context information is also needed, such as grain sizes/surface cohesion and other regolith properties, and composition (including water ice content) at sub-meter scales and below the surface. In situ age dating of young features such as gully fans would also be of value for improving interpretations, but luminescence techniques for Mars appropriate for these young ages remain unproven and challenging [56].

Coordinated collection of data related to activity and the driving environmental conditions is valuable for understanding active processes. Since such processes are by definition time variable, asynchronous data collection can yield ambiguous results. For instance, temperature data from the Thermal Emission Imaging System (THEMIS) have been used to constrain RSL water contents [57], but simultaneous HiRISE observations of the existence and size of the RSL did not exist, making the interpretation ambiguous [58]. The same challenge could recur for SAR observations of RSL [52] or other candidate aqueous features.

Many useful observations can be made from orbit, and orbital instruments are presently the only way to obtain planet-wide tracking of surface changes and environmental conditions. However, surface investigations are generally needed to fully decipher the processes and local environments. The most useful constraints on process models would come from landed investigations capable of directly observing processes in action and correlating them with simultaneous data on local environmental conditions. The major challenges are (1) the need for precision landing or mobility in order to place instruments in a location suitable for close study, which for active processes are often on steep slopes or inaccessible terrains, and (2) in some cases, the need to survive extreme conditions such as winter night, low temperatures, and seasonal frost deposition [28]. Long-range surface mobility thus represents an enabling technology for studies of active processes. This is particularly the case for processes such as RSL and gully flows, which are localized on steep slopes. The Mars Science Helicopter concept [59] represents one possible way to achieve this mobility and conduct measurements of the planetary boundary layer, but other aerial concepts also exist. Environmental measurements could also be achieved by networks of small stations without mobility or precision landing but would be less directly linked to surface changes if they are not closely coupled with observations of surface changes at the same site. Processes associated with seasonal $\mathrm{CO}_{2}$ frost are important for many forms of activity but have never been investigated at the surface [55]; mission concepts to conduct such studies have been considered by several community studies $[28,51]$. 


\section{Conclusion}

Mars is an active world, with ongoing surface processes that can be studied from the surface and from orbit via a range of mission architectures. Observations of these changes are revealing new processes that must be studied on Mars to be understood. The implications of these processes reach back in time to affect all areas of Mars science and forward to further robotic and human exploration and are a high scientific priority for the next decade. The theme has strong international interest and could represent an arc of missions as discussed by [60].

\section{References}

[1] McEwen A. S. et al. (2011) Science, 333, 740-743. [2] Dundas C. M. et al. (2017) Nature Geosci., 10, 903-907. [3] Malin M. C. \& Edgett K. S. (2000) Science, 288, 2330-2335. [4] Malin M. C. et al. (2006) Science, 314, 1573-157. [5] Costard F. et al. (2002) Science, 295, 110-113. [6] Christensen P. R. (2003) Nature, 422, 45-48. [7] Dundas C. M. et al. (2019) GSL Spec. Pub., 467, 67-94. [8] Diniega S. et al. (2013) Icarus, 225, 526-537. [9] Sullivan R. et al. (2001) JGR, 106, 23,607-23,633. [10] Bhardwaj A. et al. (2019) Rev. Geophys., 57. [11] Heyer T. et al. (2018) Planet. Space Sci., 159, 56-65. [12] Dundas C. M. et al. (2019) GRL, 46, 5075-5082. [13] Russell P. S. et al. (2008) GRL, 35, L23204. [14] Fanara L. et al. (2020) Icarus, 342, article \#113434. [15] Leighton R. B. \& Murray B. C. (1966) Science, 153, 136-144. [16] Malin M. C. \& Edgett K. S. (2001) JGR, 106, 23,429-23,570. [17] Piqueux S. et al. (2003) JGR, 108(E8):3-1. [18] Kieffer H. H. et al. (2006) Nature, 442, 793-796. [19] Portyankina G. et al. (2017) Icarus, 282, 93-103. [20] Hansen C. J. et al. (2015) Icarus, 251, 264-274. [21] Malin M. C. et al. (2001) Science, 294, 2,1462,148. [22] Buhler P. et al. (2020) Nature Astron., 4, 364-371. [23] Byrne S. et al. (2008) Planet. Space Sci., 56, 194-211. [24] Byrne S. et al. (2009) Science, 325, 1674-1676. [25] Dundas C. M. et al. (2018) Science, 359, 199-201. [26] Mellon M. T. et al. (2009) JGR, 114, E00E06. [27] Mellon M. T. et al. (2009) JGR, 114, E00E07. [28] ICE-SAG Report (2019), 157 pages posted at http://mepag.nasa.gov/reports.cfm. [29] Daubar I. J et al. (2013) Icarus, 225, 506-516. [30] Daubar I. J. et al. (2019) JGR, 124, 958-969. [31] Bourke M. et al. (2008) Geomorphology, 94, 247-255. [32] Sullivan R. et al. (2008) JGR, 113, E06S07. [33] Silvestro S. et al. (2010) GRL, 37, L20203. [34] Bridges N. T. et al. (2011) Geology, 40, 31-34. [35] Ayoub F. et al. (2014) Nature Commun., 5:5096. [36] Hansen C. J. et al. (2011) Science, 331, 575-578. [37] Diniega S. et al. (2017) GSL Spec. Pub., 467, 95-114. [38] Banks M. E. et al. (2018) JGR, 123, 3,205-3,219. [39] Chojnacki M. et al. (2019) Geology, 47, 427-430. [40] Lapotre M. G. A. et al. (2016) Science, 353, 55-59. [41] Sullivan R. \& Kok J. F. (2017) JGR, 122, 2,111-2,143. [42] Lämmel M. et al. (2018) Nature Phys, 14, 759-765. [43] Geissler P. E. et al. (2016) Icarus, 278, 279-300. [44] Sagan C. et al. (1972) Icarus, 17, 346-372. [45] Whelley P. L. \& Greeley R. (2008) JGR, 113, E07002. [46] Giardini D. et al. (2020) Nature Geosci., 13, 205-212. [47] Hansen C. J. et al. (2019) LPSC 50, abstract \#2132. [48] McEwen A. S. et al. (2019) EPSC, abstract \#557. [49] Newman C. et al. (2020) Decadal Survey white paper. [50] NEX-SAG Report (2015), 77 pages posted at http://mepag.nasa.gov/reports.cfm. [51] ICE-SAG Report (2019), 157 pages posted at http://mepag.nasa.gov/reports.cfm. [52] Watzin J. \& Haltigin T. (2020) Presentation at MEPAG Meeting \#38. [53] Rummel J. D. et al. (2014) Astrobiology, 14, 887-968. [54] MEPAG (2020) D. Banfield, ed., 89 pages posted at https://mepag.jpl.nasa.gov/reports.cfm [55] Diniega S. et al. (2020) Decadal Survey white paper. [56] Cohen B. A. et al. (2019) Astrobio., 19, 1303-1314. [57] Edwards C. S. \& Piqueux S. (2016) GRL, 43, 2016GL070179. [58] Stillman D. E. et al. (2017) Icarus, 285, 195-210. [59] Bapst J. et al. (2020) Decadal Survey white paper. [60] MASWG presentation posted at https://mepag.jpl.nasa.gov/meetings.cfm?expand=vm9 